\title{
In Vitro Immune Competence of Buffaloes (Bubalus bubalis) of Different Production Potential: Effect of Heat Stress and Cortisol
}

\author{
Joydip Mukherjee, Sujata Pandita, Ruokuobeinuo Huozha, and Manju Ashutosh \\ Dairy Cattle Physiology Division, National Dairy Research Institute, Karnal, Haryana 132001, India \\ Correspondence should be addressed to Joydip Mukherjee, joy_phy@rediffmail.com
}

Received 25 July 2010; Revised 30 December 2010; Accepted 25 January 2011

Academic Editor: Sumanta Nandi

Copyright ( $) 2011$ Joydip Mukherjee et al. This is an open access article distributed under the Creative Commons Attribution License, which permits unrestricted use, distribution, and reproduction in any medium, provided the original work is properly cited.

\begin{abstract}
Twelve healthy lactating Murrah buffaloes of similar parity (3rd) between 90 and 120 days of lactation, selected from the herd of National Dairy Research Institute (Karnal, India) and maintained at managemental practices as followed at the Institute they were included in this experiment. The animals were divided into two groups based on their production level in previous lactation. The average milk production level of group 1 and II was 9.3 and 6 lit/day, respectively. Blood was collected from these buffaloes on three occasions 10 days apart. The lymphocytes were separated and cultured in RPMI 1640 medium with PHA-P for $24 \mathrm{~h}$ at $37^{\circ} \mathrm{C}$ in a humidified $\mathrm{CO}_{2}$ incubator $\left(95 \%\right.$ air and $5 \% \mathrm{CO}_{2}$ ). The lymphocyte responsiveness was also evaluated in response to the in vivo heat stress and in vitro cortisol. Mitogen-induced stimulation index was not affected by production level $(P<.01)$. Stimulation index was significantly reduced $(P<.01)$ in both the groups when cortisol was added at $2.0 \mathrm{ng}$ level in the culture. However, in heat-stressed buffaloes stimulation index did not vary despite increasing levels of cortisol, thus indicating that lymphocyte may become cortisol resistant during periods of acute heat stress. The results showed that lymphocyte proliferation response can be effectively used to study buffalo cell-mediated immunity in vitro.
\end{abstract}

\section{Introduction}

Induction of lymphocyte proliferative response induced by antigen/mitogen in vitro has been shown to be representative of cellular immunocompetence. This measure can potentially be used as an indicator of an individual's ability to mount an immune response to specific pathogen or immunomodulators. A series of in vitro studies in dairy cattle have demonstrated that exposure of bovine peripheral blood mononuclear cells to short and severe heat shock reduced their responsiveness to mitogens or decreased the number of viable cells $[1,2]$. These authors suggested that exposure to high temperature in vitro can depress responses of lymphocytes; apparent adaptive mechanisms induced by in vivo heat stress provide protection from effects of high temperature seen in vitro. But so far no studies on validation have been conducted on the in vitro immune competence of buffaloes in response to heat stress. Therefore the present study has been designed to evaluate the potential of using in vitro lymphocyte proliferation assay for determining the cellular immunocompetence of lactating buffaloes in response to in vivo heat stress and in vitro cortisol.

\section{Materials and Methods}

Twelve lactating Murrah buffaloes, apparently healthy and of similar parity (3rd) between 90 and 120 days of lactation were selected from the herd of National Dairy Research Institute(Karnal, India) and were divided into two groups according to milk production during previous lactation that is, average lactation yield $\pm 1 \mathrm{SD}$. Based on this, the production average of group I and II corresponded to 9.33 and 6.0 lit/day, respectively. All these buffaloes were maintained under general managemental practices as followed at the institute. The feed and water were available ad lib.

Buffaloes of both the groups were used as lymphocyte donors. Blood ( $15 \mathrm{~mL} /$ buffalo) was drawn in sterile heparinised vacutainer tube from jugular vein puncture, posing minimum disturbance to the animal during collection, on three occasions 10 days apart, at $6.00 \mathrm{AM}$ in the morning. 
Buffaloes of high yielding groups were also exposed to acute heat stress $\left(45^{\circ} \mathrm{C}, 55 \% \mathrm{RH}\right)$ for $3 \mathrm{~h}$. The blood samples were collected before exposure and after $3 \mathrm{~h}$ of exposure from these heat exposed buffaloes $(n=6)$. Immediately after collection the samples were transported to the laboratory in ice for further processing.

Blood was centrifuged at $3000 \mathrm{rpm}$ for $30 \mathrm{~min}$; the buffy coat was harvested and resuspended in 1:1 v/v Dulbeccos Phosphate Buffer Saline (DPBS). The total contents were carefully layered on lymphocyte separation medium (Histopaque 1077) at concentration of $4: 1 \mathrm{v} / \mathrm{v}$ in sterile $15 \mathrm{~mL}$ polypropylene centrifuge tube and centrifuged at $2500 \mathrm{rpm}$ for 30 minutes at room temperature. The lymphocyte rich layer was removed and washed twice with DPBS. The contents were centrifuged at 1200-1500 rpm for $15 \mathrm{~min}$. each time washing with DPBS. Contaminating erythrocytes were then lysed with addition of few drops of distilled water and again washed with DPBS. The washed cells were then resuspended in RPMI 1640 media (3-4 mL) supplemented with antibiotics and 10\% FCS and centrifuged at 1200-1500 rpm for $15 \mathrm{~min}$. After the final wash, the lymphocyte rich pellet was resuspended in $5 \mathrm{~mL}$ of the culture medium.

Trypan blue exclusion method was used to determine the proportion of viable cells in the separated lymphocytes.

The lymphocyte suspension was adjusted to $5 \times 10^{6}$ live lymphocytes/mL by the culture media (RPMI 1640) containing $10 \%$ FCS. $200 \mu \mathrm{L}$ of the diluted cell suspension per well in triplicate was placed in a 96 well flat bottomed tissue culture plate. The mitogen employed in the present study was phytohemagglutinin (PHA-P) at the concentration of $5 \mu \mathrm{g} / \mathrm{mL}$ of the final culture volume $(200 \mu \mathrm{L})$, a concentration that had been determined previously to provide maximal stimulation of bovine lymphocyte [3]. The cells were allowed to proliferate with and without mitogen (PHA-P) to determine the difference between cell proliferations. Cortisol, dissolved in culture media was tested at concentrations of $0,0.5,2$, and $10 \mathrm{ng} / \mathrm{mL}$. All cultures were allowed to incubate at $37^{\circ} \mathrm{C}$ in a humidified $\mathrm{CO}_{2}$ incubator $\left(95 \%\right.$ air and $5 \% \mathrm{CO}_{2}$ ) for $24 \mathrm{~h}$. The proliferative response of lymphocyte was estimated using the colorimetric MTT (tetrazolium) according to the procedure given by Mosmann [4]. Lymphocyte blastogenic response was expressed as stimulation index (SI) and was calculated as follows.

$$
\text { Stimulation Index }(\mathrm{SI})=\frac{\mathrm{OD} \text { of the stimulated cells }}{\text { OD of the unstimulated cells }} \text {, }
$$

where OD Optical Density at $503 \mathrm{~nm}$ with reference to $630 \mathrm{~nm}$. All analyses was done using Windstat 8.0 software package. Data from different experiments are presented as mean \pm SE. Analysis of variance of the data was done using RBD factorial design to see the effect of milk production and different levels of cortisol on lymphocyte blastogenesis. The responses have been compared with respect to unstimulated cells for different experiment involving the mitogen and cortisol. The interaction effects including animals $\times$ group, group $\times$ treatment, animals $\times$ treatment $\times$ group were also calculated. To see the effect of in vivo heat stress and in vitro cortisol treatment on lymphocyte blastogenesis effect of heat stress, effect of cortisol and interaction effect (group $\times$ treatment) were calculated.

\section{Results}

Mitogen-induced stimulation index in buffaloes exhibiting different levels of milk production have been presented in Figure 1. Mitogen-induced lymphocyte blastogenesis was significantly $(P<.01)$ different in different buffaloes in both the groups. There were no significant differences between the groups. The variations observed were independent of milk production potential of these buffaloes.

Mitogen-induced stimulation index at different levels of cortisol in different groups of buffaloes have been presented in Figure 2. The stimulation indices in group I were significantly different $(P<.01)$ between cortisol treatments. The interaction effects of group $\times$ treatment $(P<.01)$ were also significant. The stimulation index of group II tended to differ significantly between treatments $(P<.05)$. The interaction effect as mentioned earlier was also significant $(P<.001)$ for this group. But, when the two groups were compared with respect to blastogenic response, the differences were not found to be significant. This indicated that the animals of both the groups did not react identically to different levels of cortisol. While cortisol at the level of $0.5 \mathrm{ng} / \mathrm{mL}$ did not affect the lymphocyte blastogenesis, it significantly reduced blastogenesis at $2 \mathrm{ng} / \mathrm{mL}$ in both the groups. Due to animal variations, the responses at different levels of cortisol supplementation were not found to be linear.

The blastogenic response was further tested in buffaloes of group I $(n=6)$ in response to imposition of in vivo acute heat stress and in vitro cortisol (Figure 3 ). There was significant $(P<.01)$ decrease in lymphocyte blastigenic response after exposure of heat stress. The stimulation index prior to exposure was significantly different between treatments $(P<.01)$. During pre-exposure the lymphocyte blastogenesis reduced significantly $(P<.01)$ at 2 ng level of cortisol and remain unaltered with the increasing levels of cortisol. After the exposure of acute heat stress the lymphocyte blastogenesis followed similar trends as pre exposure therefore, it indicated that upon exposure to heat stress the lymphocytes become either nonresponsive or cortisol resistant and therefore, do not respond to exogneous cortisol (Figure 4).

\section{Discussion}

Lymphocyte stimulation is widely used to measure (i) immune competence by stimulation of lymphocytes with phytomitogens $[5,6]$ (ii) histocompatbility by mixed leukocyte culture [7] and (iii) exposure to infectious agents by stimulation of lymphocytes with specific antigens [8]. Zimmerman et al. [9] associated the mitogen stimulated lymphocyte blastogenesis to host immunosuppression induced by parasites in sheep. In this investigation, cell-mediated immunity was assessed by measuring the proliferation of mitogenstimulated lymphocytes in buffaloes under different experiments. The response was not found to be related to milk 


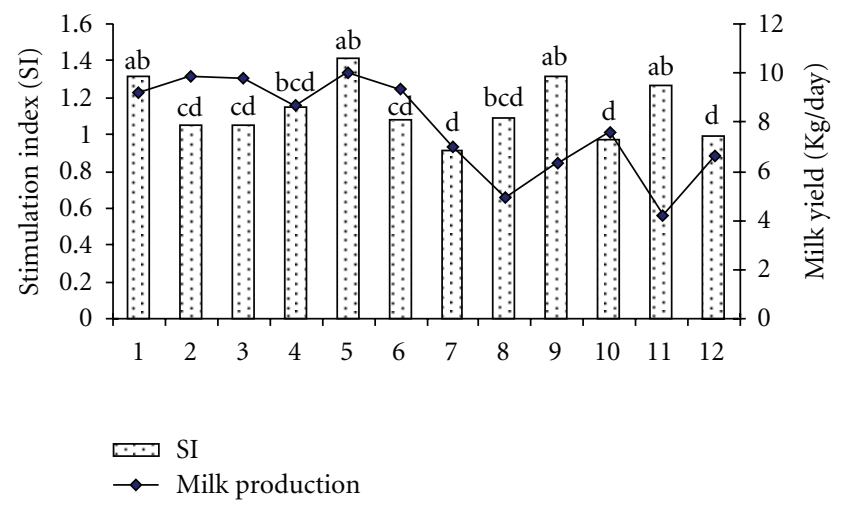

FIGURE 1: Mitogen-induced stimulation index in buffaloes exhibiting different levels of milk production. Values are mean \pm SEM. Bars with values in different groups that lack a common letter are significantly different $(P<.05)$.

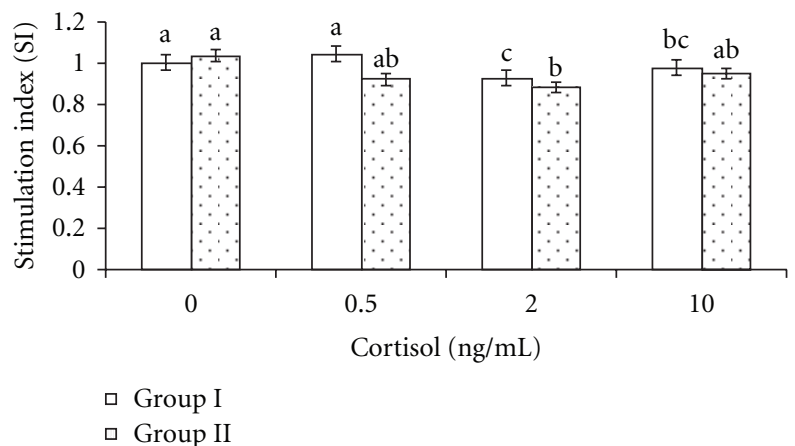

Figure 2: Mitogen-induced stimulation index at different levels of cortisol in different groups of buffaloes. Values are mean \pm SEM. Bars with values in different groups that lack a common letter are significantly different $(P<.05)$.

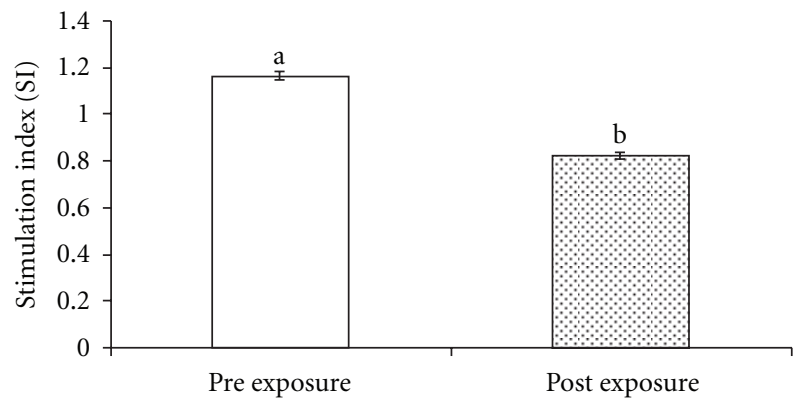

FIGURE 3: Mitogen-induced stimulation index in buffaloes after exposed to acute heat stress. Values are mean \pm SEM. Bars with values in different groups that lack a common letter are significantly different $(P<.05)$.

production potential of these buffaloes. This was realized from the fact that rate of lymphocyte blastogenesis was similar in buffaloes having a potential of 6.0 and 9.33 lit/day. Variations in the rate of blastogenesis from animal to animal have also been previously reported for outbred populations of cattle and for lactating Holsteins [10]. These variations have also been reported to be influenced by season and the age of the bovine [3].

Glucocorticoids are suppressive to a variety of lymphocyte functions. During the past several years, a number of

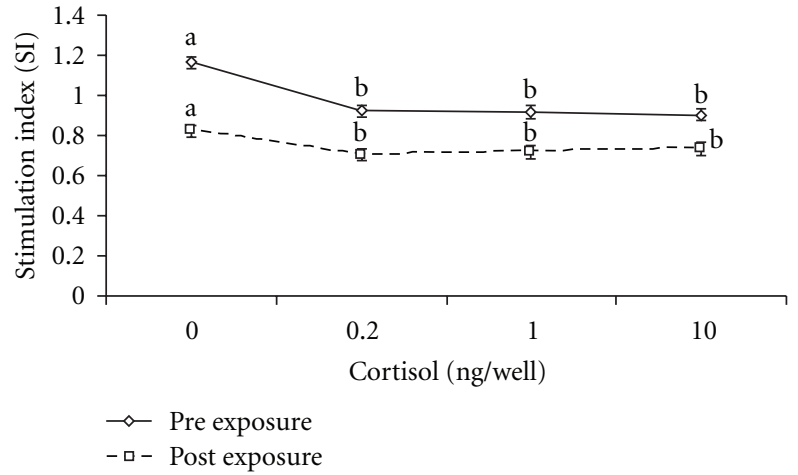

FIgURE 4: Stimulation index at different levels of cortisol in heat stressed buffaloes. Values are mean \pm SEM. Bars with values in different groups that lack a common letter are significantly different $(P<05)$.

studies have indicated that the glucocorticoids are inhibitory to lymphocyte functions in vitro when administered at pharmacological levels [11-15]. Segel et al. [16] found inhibition of human lymphocyte blastogenesis by cortisol only when suboptimal PHA levels were used. Chenault, [17] demonstrated that increasing concentration of cortisol inhibited Con-A-induced blastogenesis such that from 10 to $10^{4} \mathrm{nM}$ little reactivity could be measured in Holstein cattle, 
but did not affect blastogenesis when PHA-P was used in place of Con-A. In the present study, cortisol at the level of $0.5 \mathrm{ng} / \mathrm{mL}$, however, did not affect the PHA-P induced lymphocyte blastogenesis; it significantly reduced blastogenesis at $2 \mathrm{ng} / \mathrm{mL}$ in both the groups of buffaloes.

Mitogen-induced lymphocyte blastogenesis upon exposure to acute heat stress was significantly reduced in this investigation. A series of in vitro studies have demonstrated that exposure of bovine peripheral blood mononuclear cells to short and severe heat shock reduce responsiveness to mitogens or decrease the number of viable cells $[1,2]$. Lacetera et al. [18] reported that different incubation temperatures simulating hyperthermia impaired DNA synthesis in mitogen-stimulated PBMC in vitro. Studies conducted in vivo have provided contradictory results regarding the effects of air temperatures above upper critical temperature on immune cell functions in bovine. Some authors reported an improvement [3] some described impairment [1, 2] and others indicated no effects [19] of heat exposure on lymphocyte functions. The large variety of experimental conditions (e.g., in vivo versus in vitro, field versus controlled environment, animals from different breeds or different ages, duration and intensity of heat exposure, lymphocyte function studied) likely explains the discrepancy among results. The differential effects of heat on proliferative response of mitogen-stimulated PBMC from Brown Swiss and Holstein cows. Lacetera et al. [18] further suggested that body temperature increase under heat-stress conditions might not be associated with the same degree of alteration of immune cell efficiency in two breeds. Generalization of this concept indicates that impairment of cell functions may start at a different level of body hyperthermia in different animals as seen in this study also. Further, prolonged exposure to severe heat stress [18] is responsible for a decline of immune cells' reactivity, which may contribute to the higher occurrence of some infections during summer [20].

\section{Conclusion}

Major findings of the present investigation permitted us to conclude that lymphocyte proliferation assay could be used as one of the measures to determine cell-mediated immunity of the Murrah buffaloes in vitro. The stimulation index significantly differed between animals, albeit not affected by production levels. stimulation index was affected by in vitro cortisol. The blastogenesis was reduced significantly at $2.0 \mathrm{ng}$ level. Mitogen-induced lymphocyte proliferation response significantly decreased in buffaloes when exposed to heat stress. However, addition of cortisol at different levels in vitro produced no further change in lymphocyte proliferation response therefore, indicating that upon exposure to heat stress the lymphocytes may become cortisol resistant.

\section{References}

[1] F. Elvinger, P. J. Hansen, and R. P. Natzke, "Modulation of function of bovine polymorphonuclear leukocytes and lymphocytes by high temperature in vitro and in vivo," American
Journal of Veterinary Research, vol. 52, no. 10, pp. 1692-1698, 1991.

[2] L. A. Kamwanja, C. C. Chase, J. A. Gutierrez et al., "Responses of bovine lymphocytes to heat shock as modified by breed and antioxidant status," Journal of Animal Science, vol. 72, no. 2, pp. 438-444, 1994.

[3] F. F. Soper, C. C. Muscoplat, and D. W. Johnson, "In vitro stimulation of bovine peripheral blood lymphocytes: analysis of variation of lymphocyte blastogenic response in normal dairy cattle," American Journal of Veterinary Research, vol. 39, no. 6, pp. 1039-1042, 1978.

[4] T. Mosmann, "Rapid colorimetric assay for cellular growth and survival: application to proliferation and cytotoxicity assays," Journal of Immunological Methods, vol. 65, no. 1-2, pp. 55-63, 1983.

[5] S. D. Douglas, "Electron microscopic and functional aspects of human lymphocyte response to mitogens," Transplantation Reviews, vol. 11, pp. 39-59, 1972.

[6] K. A. Weigel, A. E. Pneman, J. R. Thurston Jr., M. J. Stear, and D. H. Kelley, "Association of class I bovine lymphocyte antigen complex alleles with health and production traits in dairy cattle," Journal of Dairy Science, vol. 73, no. 9, pp. 25382546, 1990.

[7] E. Thorsby, "The human major histocompatibility system," Transplantation Reviews, vol. 18, pp. 51-129, 1974.

[8] F. Daguillard, "Immunologic significance of in vitro lymphocyte responses," Medical Clinics of North America, vol. 56, no. 2, pp. 293-303, 1972.

[9] G. L. Zimmerman, N. I. Kerkvliet, J. A. Brauner, and J. E. Cerro, "Modulation of host immune responses by Fasciola hepatica: responses of peripheral lymphocytes to mitogens during liver fluke infections of sheep," Journal of Parasitology, vol. 69, no. 3, pp. 473-477, 1983.

[10] F. A. Murray, E. C. Segerson, and F. T. Brown, "Suppression of lymphocytes in vitro by porcine uterine secretory protein," Biology of Reproduction, vol. 19, no. 1, pp. 15-25, 1978.

[11] T. Mori, H. Kobayashi, and T. Nishimura, "Inhibitory effect of progesterone on the phytohaemagglutinin induced transformation of human peripheral lymphocytes," Immunological Communications, vol. 4, no. 6, pp. 519-527, 1975.

[12] J. Carter, "The effect of progesterone, oestradiol and HCG on cell mediated immunity in pregnant mice," Journal of Reproduction and Fertility, vol. 46, no. 1, pp. 211-216, 1976.

[13] P. K. Siiteri, F. Febres, L. E. Clemens, J. Chang, B. Gondos, and D. Stites, "Progesterone and maintenance of pregnancy: is progesterone nature's immunosuppressant?" Annals of the New York Academy of Sciences, vol. 286, pp. 384-396, 1977.

[14] C. Pavia, P. K. Siiteri, J. D. Perlman, and D. P. Stites, "Suppression of murine allogeneic cell interactions by sex hormones," Journal of Reproductive Immunology, vol. 1, no. 1, pp. 33-38, 1979.

[15] F. A. Kincl and L. A. Ciaccio, "Suppression of immune responses by progesterone," Endocrinologia Experimentalis, vol. 14, no. 1, pp. 27-33, 1980.

[16] G. B. Segel, A. Lukacher, B. R. Gordon, and M. A. Lichtman, "Glucocorticoid suppression of human lymphocyte DNA synthesis: influence of phytohemagglutinin concentration," Journal of Laboratory and Clinical Medicine, vol. 95, no. 4, pp. 624-632, 1980.

[17] J. R. Chenault, "Steroid metabolism by the early bovine conceptus. I. $5 \beta$-reduction of neural C-steroids," Journal of Steroid Biochemistry, vol. 13, no. 5, pp. 499-506, 1980. 
[18] N. Lacetera, U. Bernabucci, D. Scalla, L. Basiricò, P. Morera, and A. Nardone, "Heat stress elicits different responses in peripheral blood mononuclear cells from Brown Swiss and Holstein cows," Journal of Dairy Science, vol. 89, no. 12, pp. 4606-4612, 2006.

[19] N. Lacetera, U. Bernabucci, B. Ronchi, D. Scalia, and A. Nardone, "Moderate summer heat stress does not modify immunological parameters of Holstein dairy cows," International Journal of Biometeorology, vol. 46, no. 1, pp. 33-37, 2002.

[20] N. B. Cook, T. B. Bennett, K. M. Emery, and K. V. Nordlund, "Monitoring nonlactating cow intramammary infection dynamics using DHI somatic cell count data," Journal of Dairy Science, vol. 85, no. 5, pp. 1119-1126, 2002. 

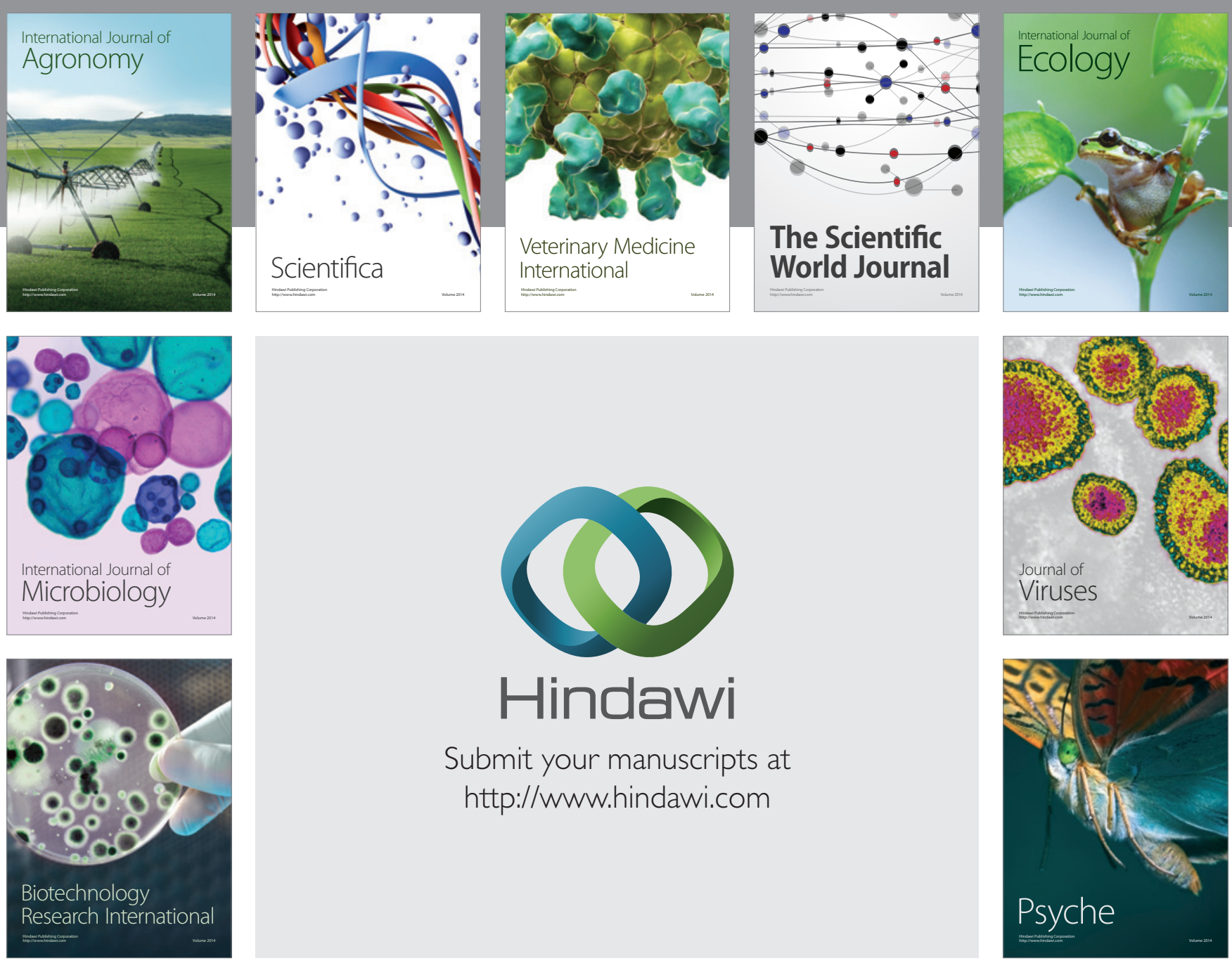

Submit your manuscripts at

http://www.hindawi.com
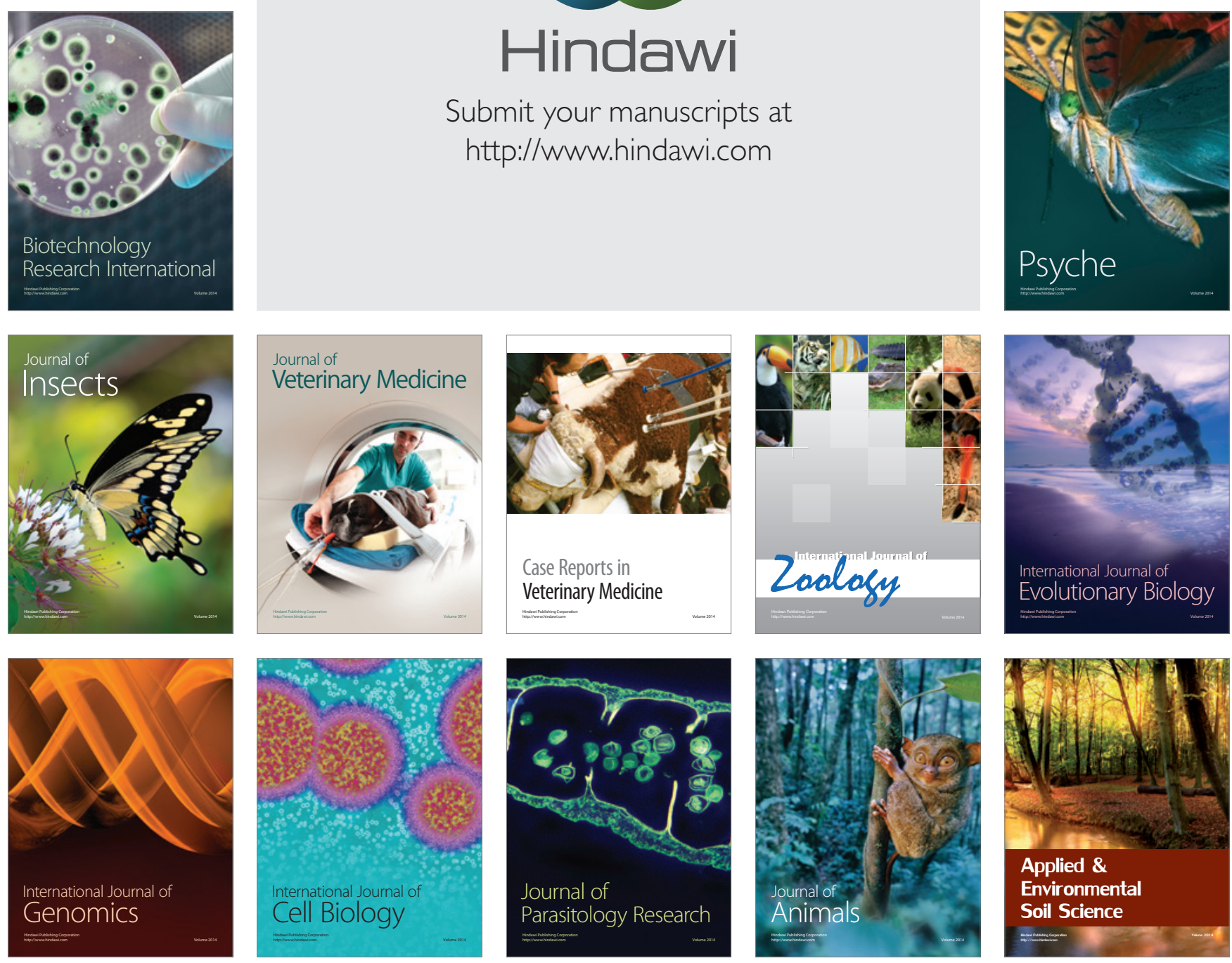